\title{
Competing effects on the average age of infant death
}

\author{
Monica Alexander* Leslie Root ${ }^{\dagger}$
}

\begin{abstract}
In recent decades, the relationship between the average length of life for those who die in the first year of life - the lifetable quantity ${ }_{1} a_{0}$ - and the level of infant mortality, on which its calculation is often based, has broken down. The very low levels of infant mortality in the developed world correspond to a range of ${ }_{1} a_{0}$ quantities. We illustrate the competing effect of falling mortality and reduction in preterm births on ${ }_{1} a_{0}$, through two populations with very different levels of premature birth - infants born to non-Hispanic white mothers and to non-Hispanic black mothers in the United States, using linked birth and infant death cohort data. Through simulation, we further demonstrate that falling mortality reduces ${ }_{1} a_{0}$, while a reduction in premature births increases it. We use these observations to motivate the formulation of a new approximation formula for ${ }_{1} a_{0}$ in low-mortality contexts, which aims to incorporate differences in preterm birth through a proxy measure - the ratio of infant to under-five mortality. Models are built and tested using data from the Human Mortality Database. Model results and validation show that the newly proposed model outperforms existing alternatives. Keywords: Infant mortality, prematurity, life table.
\end{abstract}

\footnotetext{
${ }^{*}$ Departments of Statistical Sciences and Sociology, University of Toronto. monica.alexander@utoronto.ca.

${ }^{\dagger}$ Institute of Behavioral Science, University of Colorado Boulder. leslie.root-1@colorado.edu. Both authors contributed equally to this work.
} 


\section{Introduction}

The average length of life for those who die in the first year of life, ${ }_{1} a_{0}$, is an important lifetable quantity, the first building block of the calculation of person-years lived that ultimately sums to the expectation of life at birth. However, the data required to calculate ${ }_{1} a_{0}$ exactly are often not readily available, and as such, producers of lifetables usually rely on empirical relationships between the overall level of infant mortality and average age at death to calculate an approximate ${ }_{1} a_{0}$. The most common of these relationships, the Coale-Demeny and Keyfitz-Flieger formulas, rely on the general rule that as infant mortality falls, deaths become increasingly concentrated early in the interval, so ${ }_{1} a_{0}$ also falls (Coale, Demeny, and Vaughan 1983; Keyfitz and Flieger 1971). The Keyfitz-Flieger formula, for example, which is referenced in central demographic textbooks (e.g. Wachter 2014), is ${ }_{1} a_{0}=0.07+1.7_{1} M_{0}$, where ${ }_{1} M_{0}$ is the infant mortality rate.

This relationship can be explained with reference to Bourgeois-Pichat's theory (1951a, 1951b), which assumes all infant deaths can be categorized as arising from either endogenous or exogenous causes, with the latter tending to occur later in the first year. As overall mortality goes down, exogenous causes are increasingly eliminated, and the distribution of infant deaths shifts towards the endogenous causes, which are more likely to cause death relatively soon after birth.

However, at the very low levels of infant mortality currently observed in the developed world, the monotonic relationship between ${ }_{1} a_{0}$ and infant mortality is no longer the case, and in fact, ${ }_{1} a_{0}$ has been rising in many countries since the 1970s. Andreev and Kingkade (2015) attribute this shift to medical advances that reduce very early deaths due to congenital conditions and conditions of prematurity. Furthermore, they note that the relationship between

level of mortality and ${ }_{1} a_{0}$ becomes comparatively weak at these low levels of mortality. In this paper, we investigate the reason for this, and illustrate two competing effects on ${ }_{1} a_{0}$ : the overall level of infant mortality, and the share of births that are premature. 
Recent work on age patterns in early-life mortality has not taken premature birth into consideration as a determinant of either the level or the shape of infant mortality (e.g. MejíaGuevara and Tuljapurkar 2019; Mejía-Guevara et al. 2019; Galley and Woods 1999). This is no doubt partly because of the lack of availability of data on premature birth (Blencowe et al. 2012). However, premature birth is clearly important in describing infant mortality patterns; despite advances, conditions of prematurity still play an outsize role in infant death (Callaghan et al. 2006). And because prematurity is difficult to predict and prevent, and is correlated with a number of individual-level factors including maternal age, health and behavior, as well as socioeconomic status and race, it may vary substantially both among and within populations with relatively low infant mortality (Tucker and McGuire 2004; Purisch and Gyamfi-Bannerman 2017). These two facts taken together indicate that an ideal model of infant mortality would include information on the preterm birth rate.

In this paper, we first illustrate the competing effect of mortality and prematurity rates on the average age of infant death, ${ }_{1} a_{0}$, through an example using data from two U.S. populations with very different levels of premature birth - infants born to non-Hispanic white mothers and infants born to non-Hispanic black mothers. We then perform a simulation exercise, calculating ${ }_{1} a_{0}$ at a wide range of mortality and prematurity levels, to further illustrate these competing effects. We then utilize these observations in developing a new formula for ${ }_{1} a_{0}$, which aims to account for not only the level of infant mortality, but also the level of prematurity, using as a proxy the ratio of infant to under-five mortality. Our proposed approximation approach outperforms existing alternatives and provides new insight into understanding patterns of the average age of infant death in low-mortality settings. 


\section{The relationship between ${ }_{1} a_{0}$, infant mortality, and prematurity}

Although infant mortality relates to all deaths in the first year, the distribution of these deaths over time is far from uniform. Figure 1 shows the distribution of infant deaths in the United States in 2012. The distributions have been plotted separately for pre- and full-term

births, where preterm births are defined as those occurring before a gestational age of 37 full weeks from the last menstrual period.

Irrespective of the prematurity of births, the largest share of infant deaths occurs in the first several days. However, as Figure 1 shows, the distribution is particularly skewed and concentrated for preterm births, with over $60 \%$ of infant deaths occurring within the first five days.

[Figure 1 about here.]

In terms of the relationship between overall infant mortality, prematurity and the distribution of death times, the following general observations can be made:

1. The distribution of the timing of infant deaths is left skewed, with the majority of deaths in the first few days (Figure 1 above).

2. All other things being equal, the degree of this left skewness increases as mortality decreases.

3. The distribution of the timing of infant deaths conditional on births being premature is also heavily left skewed, with an even larger density of deaths in the first few days (Figure 1 above).

4. The share of births that are premature tends to decrease as mortality decreases (WHO 2018). 
Why does the relationship between ${ }_{1} a_{0}$ and infant mortality become unclear at lower mortality levels? Because statements 1 \& 2 above have the opposite effect from statements 3 \& 4 on ${ }_{1} a_{0}$. As overall mortality conditions improve, we expect that exogenous causes of death that occur later in the first year of life decrease, and so ${ }_{1} a_{0}$ will decrease. However, as overall mortality conditions improve, we also expect the share of births that are premature to decrease, and so ${ }_{1} a_{0}$ will increase. These observations imply that trends in ${ }_{1} a_{0}$ over time may go up or down; and that the degree of similarity between populations' infant mortality rates does not necessarily imply any particular degree of similarity between their ${ }_{1} a_{0}$ values (and vice versa).

These competing effects are illustrated in this section; first, by examining infants deaths in the United States by race, and second, by simulating the effects on ${ }_{1} a_{0}$ of changes in overall mortality risk and the prevalence of prematurity.

\subsection{Example: U.S. infant deaths by race}

Racial disparities in U.S. infant mortality are longstanding and well-known. Although mortality has fallen for all racial and ethnic groups, babies born to non-Hispanic black mothers have for over thirty years consistently died at more than twice the rate of those born to non-Hispanic white mothers (Ely and Driscoll 2019; Hummer et al. 1999; Mathews, MacDorman, and Menacker 2013). Given this substantial inequality, it could be assumed that the average age of infant death, ${ }_{1} a_{0}$, would be noticeably higher for the black population than the white population. However, as illustrated in this section, this is not the case. The racial disparity in infant mortality is not simply one of magnitude; patterns of preterm birth, low birth weight, age at death and cause of death all differ substantially between these two groups (Bediako, BeLue, and Hillemeier 2015; Ely and Driscoll 2019; MacDorman 2011; Riddell, Harper, and Kaufman 2017). Indeed, it is in part because of higher rates of preterm birth that infants born to non-Hispanic black mothers die so much more frequently. 
These high rates of prematurity, in turn, are caused not only by broad and well-documented socioeconomic disparities between black and white populations, but by the health effects on black mothers of lifelong exposure to racism, and by medical racism experienced during pregnancy (Kramer and Hogue 2009; Alhusen et al. 2016). The interaction of these factors causes surprising patterns in ${ }_{1} a_{0}$.

\subsubsection{Data}

Data on deaths in the first year of life for infants born to non-Hispanic black (NHB) and non-Hispanic white (NHW) mothers comes from the National Bureau of Economic Research collection of U.S. Birth Cohort Linked Birth and Infant Death Data of the National Center for Health Statistics' National Vital Statistics System, years 2008-2012 (NBER 2020). Data on live birth counts for the same years, by gestational age, mother's race, and sex, comes from the Center for Disease Control and Prevention's publicly-available WONDER database (Centers for Disease Control and Prevention National Center for Health Statistics 2020). Cohort prematurity rates were calculated as the number of premature births divided by the total number of live births, and infant mortality rates were calculated as the number of deaths divided by the number of live births. Mortality was calculated according to race, birth cohort, premature status, and age at death. In line with WHO classification, premature status was grouped into 4 categories based on the last menstrual period (LMP) measure of gestational age: extremely preterm (born at $<28$ full weeks of gestation), very preterm (28 to $<32$ full weeks of gestation), later preterm (32 to $<37$ full weeks of gestation) and full-term (37 full weeks of gestation or more) (WHO 2018). Age at death was split into first-week $(<7$ days old at death), neonatal ( $<28$ days; includes first-week deaths), and post-neonatal (28 days or older at death). An average of 3021 births (0.1\% of the total) and 147 deaths (0.8\% of the total) were excluded each year due to missing gestational age data. 


\subsubsection{Mortality, prematurity, and average age at death}

Infant mortality rates for NHB and NHW populations in 2012, stratified by age at death and prematurity of birth, are shown below (Table 1). ${ }^{1}$

Large differences are observed in mortality rates by race that are not stratified by prematurity. Overall mortality is 2.21 times higher for infants born to NHB mothers than for those born to NHW mothers, at 11.00 per thousand versus 4.98 per thousand. When stratifying by age at death, a gradient is evident; racial ratios for mortality are highest in the first week (2.29), somewhat lower for neonatal mortality (2.24), and lowest, though still above 2, for post-neonatal mortality (2.15).

Among premature infants, mortality rates by gestational age are similar for those born to NHB and NHW mothers. For the extremely preterm, the ratio of the NHB mortality rate to the NHW mortality rate is 0.99 . For the very preterm, the ratio is 1.00 , and for later preterm infants, it is 1.04. For full-term births, however, NHB mortality is 1.70 times higher than NHW mortality.

[Table 1 about here.]

This mismatch between racial patterns of mortality by gestational age and racial patterns by age at death is compositional; it is explained by a large difference in the distribution of births by gestational age, shown in Table 2. Fewer infants born to NHB mothers are born at full term, and among those born early, they are more likely than those born to NHW mothers to be extremely or very preterm. Because mortality risk drops rapidly with gestational age, this means that a larger share of infants born to NHB mothers are at high risk of dying.

[Table 2 about here.]

\footnotetext{
${ }^{1}$ Because there is no large time trend in racial difference - that is, infant mortality is declining in a similar way for both racial groups over this period - figures are given for the most recent birth cohort for which data are available (2012). Figures for four previous cohorts may be found in Appendix A. Similarly, although ${ }_{1} a_{0}$, as a life table quantity, is usually calculated by sex, sex differences are not material to the patterns of prematurity and mortality by race and are thus not included here.
} 
This compositional difference, in turn, leads to an instance of Simpson's paradox in the average age of infant death, wherein a trend observed in aggregate reverses when data are decomposed into subgroups. In aggregate, infants born to NHB mothers have a slightly lower average age at death, 40.87 days versus 45.29 days for infants of NHW mothers. This is somewhat surprising, given NHB infants' higher mortality: as described above, a shorter ${ }_{1} a_{0}$ is generally associated with lower overall mortality and a lower share of preventable mortality, as deaths later in infancy are more likely to be caused by external factors and treatable diseases (Andreev and Kingkade 2015).

And indeed, within each subgroup by gestational age at birth, the average age at death is higher for NHB infants, indicating a greater proportion of deaths later in infancy. The difference is marked for all premature infants, regardless of gestational age; among full-term births, NHB infants' ${ }_{1} a_{0}$ is only $3 \%$ longer than NHW infants'. (Table 3)

[Table 3 about here.]

\subsection{Illustrating the competing effects through simulation}

In order to further investigate the competing effects on ${ }_{1} a_{0}$, we performed a simulation exercise that allows us to change the overall level of mortality and prematurity independently, and then assess the consequent change in ${ }_{1} a_{0} \cdot{ }^{2}$ Specifically, we carry out two simulations:

- Scenario 1: Vary the risk of infant mortality of a population, holding the rate of prematurity constant.

- Scenario 2: Vary the share of premature births in a population, holding mortality risk constant.

\footnotetext{
${ }^{2}$ This independent variation is not meant to imply that levels of mortality and prematurity are independent, but only that, because other factors also contribute to a population's mortality rate, it is possible for a population at a given level of mortality to have a range of levels of premature birth, and vice versa.
} 
In order to be able to simulate plausible times of infant deaths, we need a suitable expression for the distribution of infant deaths. As illustrated in Figure 1, the distribution of infant deaths at the low levels of mortality we are interested in is highly skewed, with a large proportion of deaths occurring in the first week. The shape of these distributions is not readily captured by any classic parametric distributions. However, we found that the shape of infant death distributions was well-captured by a piecewise constant hazard (PCH) model, with time intervals partitioned at days 1-7, 14, 28, 60, 90, 180, and 365. The PCH model assumes constant exponential hazards within each of these time intervals, which allows the model to be estimated using Poisson regression.

PCH models were fit separately to all full-term births/deaths and all preterm births/deaths using the 2012 U.S. births and deaths data. Once estimates of hazards were obtained, we simulated different sets of survival times based on varying: 1) the overall infant mortality risk factor and 2) the overall share of births that are premature. Details on the statistical model and simulation can be found in Appendix C.

The results of these simulations are shown in Figure 2. The results clearly show that changes in overall infant mortality and changes in prematurity act in opposite directions. For increases in the risk profile of mortality, the average age of infant death steadily increases until a risk factor of around 2 , when the estimated ${ }_{1} a_{0}$ plateaus. This plateau is a consequence of the shape of the death distribution, with increases in later infant mortality being balanced out by the increases in high hazards in the earlier-infant mortality. For increases in the share of premature deaths (Figure $2 b$ ), the average age of infant death steadily declines. It is worth noting that the magnitude of changes in ${ }_{1} a_{0}$ in response to changes in prematurity are larger than those based on increasing the overall risk of mortality. This is broadly consistent with the pattern observed in the U.S. data; faced with both higher rates of prematurity and a higher overall risk of mortality, NHB have a shorter ${ }_{1} a_{0}$ than NHW infants, not a longer one. Figure $2 \mathrm{c}$ combines variability across prematurity and mortality risk to illustrate changes in ${ }_{1} a_{0}$ in two dimensions. The lighter the color, the higher the value of ${ }_{1} a_{0}$. While 
${ }_{1} a_{0}$ increases monotonically with increased mortality risk, and decreases monotonically with increased prematurity, the trajectory of ${ }_{1} a_{0}$ over time depends on the relative changes across the two dimensions.

[Figure 2 about here.]

\section{A new approximation formula for ${ }_{1} a_{0}$ in low- mortality conditions}

In this section, we outline a new proposed model to estimate ${ }_{1} a_{0}$, the data we used for model building and evaluation, and our evaluation and validation strategy. In brief, we propose the ratio of infant to under-five child mortality as a proxy for the prevalence of prematurity, and include this ratio in a model for ${ }_{1} a_{0}$ along with the infant mortality rate. We compare the performance of our new proposed model to the existing best alternative, a piecewise linear model, as proposed by Andreev and Kingkade (2015), and show that it outperforms this model on several different model evaluation metrics.

\subsection{A proxy for prematurity}

We have illustrated that the rate of premature birth appears to play an important role in ${ }_{1} a_{0}$ at low rates of infant mortality, and an ideal model for calculating ${ }_{1} a_{0}$ would include information on both infant mortality and prematurity. Unfortunately, with the exception of U.S. microdata, detailed premature birth data is not widely available (Blencowe et al. 2012). As an alternative measurement, we argue that, at these low levels of mortality, the ratio of early (i.e. infant) to total (i.e. under-five) child mortality proxies the early vs. late pattern within infant mortality. That is, at a given level of infant mortality, a higher level of overall mortality for those under age five signals that a relatively larger share of infant mortality 
is due to exogenous causes, while a lower level of overall under-five mortality signals the opposite - that infant mortality is more skewed toward endogenous causes arising from premature birth. Indeed, looking at the data on infant and child mortality and prematurity in the United States by sex and race/ethnicity, there is a strong positive relationship between the ratio of infant to under-five mortality (IMR/U5MR) and the proportion of births that are premature (Figure 3). The correlation between these two indicators across all groups is 0.95 , and while the global regression equation is shown in Figure 3, the strong positive association also holds for each sex and race/ethnicity group. Thus, motivated by the United States, where data on mortality and prematurity are readily available, there is evidence to suggest the ratio of IMR to U5MR is a reasonable proxy for prematurity.

[Figure 3 about here.]

Turning to the relationship between the ratio and the average age at infant death, we can see a clear negative relationship based on available data across all countries in the Human Mortality Database (igure 4). Using the ratio as a explanatory variable within a model for ${ }_{1} a_{0}$ is advantageous as it is easily calculable for all countries based on existing data and estimates. For instance, the United Nations Inter-agency Group on Mortality Estimation (IGME) produces infant mortality and under-five mortality estimates for all member countries. ${ }^{3}$

[Figure 4 about here.]

\subsection{Proposed model}

We propose a linear model in which the ${ }_{1} a_{0}$ is related to the infant mortality rate (IMR), defined as deaths in a particular year to those under age 1 per thousand live births, and the

\footnotetext{
${ }^{3}$ Available at childmortality.org.
} 
ratio of the infant to the under-five mortality rate (U5MR), which is defined as deaths to those under age 5 in a particular year per thousand live births. ${ }^{4}$

$$
{ }_{1} a_{0_{i}}=\alpha+\beta_{1}\left(\mathrm{IMR}_{i}\right)+\beta_{2}\left(\frac{\mathrm{IMR}_{i}}{\mathrm{U}_{5 \mathrm{MR}}}\right)+\varepsilon_{i}
$$

For reference, existing models for ${ }_{1} a_{0}$ (as in Andreev and Kingkade (2015) $)^{5}$ have the form of a piecewise linear relationship with ${ }_{1} q_{0}$ with one 'cutpoint' $(\theta)$ at which the slope of the linear relation with ${ }_{1} q_{0}$ changes. $^{6}$

$$
{ }_{1} a_{0_{i}}=\alpha+\beta_{1}\left({ }_{1} q_{0_{i}}\right)+\beta_{2}\left({ }_{1} q_{0_{i}}-\theta\right)+\varepsilon_{i}
$$

One of the main advantages of the proposed model over previous models is that it explicitly accounts for the two competing effects on ${ }_{1} a_{0}$, by incorporating additional readily-available data. In contrast, existing methods model ${ }_{1} a_{0}$ as a mathematical function of infant mortality rate using a set of arbitrary splines, without taking into consideration the demographic or biological reasons for why the relationship between ${ }_{1} a_{0}$ and infant mortality may change with the level of mortality.

\subsection{Data}

This analysis draws on data compiled by the Human Mortality Database (HMD), a joint project of the University of California, Berkeley (USA), and the Max Planck Institute for

\footnotetext{
${ }^{4}$ In cohort perspective, calculated as deaths in a cohort per thousand births in that cohort, these two quantities would be equivalent to ${ }_{1} q_{0}$ and ${ }_{5} q_{0}$, the probability of dying before age 1 and the probability of dying before age 5, multiplied by 1000; period perspective complicates the picture, since it is the case for both quantities that the numerator and denominator no longer refer to precisely the same cohort of children.

${ }^{5}$ Andreev and Kingkade (2015) use the notation ${ }_{1} q_{0}$, but refer to it as the infant mortality rate; in their calculations, it is somewhat unclear whether they are using the period IMR as it is usually calculated (described above) or the probability of death.

${ }^{6}$ Note that Andreev and Kingkade (2015) define two cutpoints, the second being the level of ${ }_{1} q_{0}$ above which ${ }_{1} a_{0}$ is estimated to be constant. However, as we are focusing on low levels of mortality, we are more interested in comparisons just in the range of the first cutpoint.
} 
Demographic Research (Germany) that collects, validates, cleans and adjusts detailed data on period and cohort mortality, available online at https://www.mortality.org. It currently includes 41 countries with reliable mortality statistics. HMD data does not allow for direct observation of ${ }_{1} a_{0}$, but it can be calculated using the Lexis triangle method detailed by Andreev and Kingkade (2015), whereby the average age of infant death is the ratio of the number of deaths in the upper Lexis triangle - that is, deaths that occur under age 1 in year $\mathrm{x}+1$ to those born in year $\mathrm{x}$ - to the total number of deaths under age 1 to those born in year x. Following Andreev and Kingkade, we use the initial raw numbers of deaths by Lexis triangle in these calculations, avoiding any adjustment which could bias the estimate of ${ }_{1} a_{0}$. With some exclusions, this analysis makes use of raw Lexis triangle death data from all countries and cohorts currently available through HMD. ${ }^{7}$ Excluded are: Bulgaria (before 2009), Estonia (before 1992), and Belarus, Russia, and Ukraine (entirely), for incomplete conformity to the WHO definition of 'live birth,' which leads to an overcount of stillbirths and reduction in first-day infant mortality; Chile and Taiwan, for suspected problems with the registration of very early infant deaths; Iceland and Luxembourg, for very low numbers of infant deaths resulting in unusual ${ }_{1} a_{0}$ or mortality rates; the Netherlands (before 1950), because of data adjustments made to account for differences in the definition of live birth; Switzerland (before 1880) for irregularities implying unusual migration patterns at age zero; and Belgium (1941-1945 and 1958-1960), Israel, and Poland (entirely), for implausible mortality rates or ${ }_{1} a_{0}$ estimates. Populations whose inclusion would result in duplication of data were also excluded (e.g., because East Germany and West Germany are included separately, the aggregate data available for Germany as a whole is not included). In addition, an infant mortality cutoff of 80 per thousand for females and 95 per thousand for males was applied, as this analysis focuses on low-mortality contexts. This yielded $2852{ }_{1} a_{0}$ observations from 27 countries or areas, for cohort years ranging from 1902 to 2018, broken down by sex (1430 female observations and 1422 male). A table listing all included populations can be found

\footnotetext{
${ }^{7}$ Data downloaded May 25, 2020.
} 
in Appendix B.

\subsection{Modeling strategy}

Our primary goal is to estimate parameters in Equation 1, which we will refer to as the 'ratio model,' and compare the performance of this model to the existing best alternative proposed by Andreev and Kingkade. We estimated model parameters for both the ratio model and Equation 2, i.e., a piecewise linear model, in a Bayesian framework using a Hamiltonian Monte Carlo algorithm, implemented using the Stan programming language in R (Carpenter et al. 2017). ${ }^{8}$

To evaluate model performance, we split the available data into two separate datasets: the 'training' dataset, which comprises a random sample of $80 \%$ of all the available data, and a 'test' dataset, which comprises of the remaining $20 \%$ of data. The idea is that the estimates of the parameters in the ratio and piecewise models are obtained by fitting models on the training dataset, and then the predictive accuracy of each model is evaluated based on the test dataset. This train/test splitting technique, which is common in statistical learning methods, minimizes issues of over-fitting and better allows for the evaluation of out-of-sample fit (James et al. 2013).

Our modeling strategy introduces two comparison issues: differences in data, and differences in estimation methods. Firstly, we have at our disposal substantially more data from HMD than was available five or more years ago, which means parameter estimates for a piecewise linear, as was estimated by Andreev and Kingkade, may differ purely because of data reasons. Secondly, our choice to estimate models within a Bayesian framework using Stan also differs from Andreev and Kingkade's analysis. To account for these differences, in addition to estimating the parameters for the piecewise linear model on the training dataset, we also

\footnotetext{
${ }^{8}$ We chose to estimate models using Stan for two reasons; first, uncertainty intervals (Bayesian credible intervals) are a natural by-product of the estimation process (unlike other optimization options that are usually required to estimate the position of the cutpoints), and second, the ability to calculate approximations to the leave-one-out cross validation using Stan allows us to easily compare and evaluate models.
} 
obtain estimates on a dataset which corresponds to the data used by Andreev and Kingkade (2015) (termed the 'AK' dataset). In doing so, we show that our estimates for a piecewise linear model fit to AK data do not differ significantly from Andreev and Kingkade's estimates.

To summarize, we fit two models (the ratio model and the piecewise linear model, Equations 1 and 2). We estimated the ratio model and piecewise linear model by sex and also for both sexes combined. Both the ratio model and piecewise linear model were estimated on the training datasets (male, female and both sexes combined) and the AK dataset. All models, including the estimates presented by Andreev and Kingkade (2015), were then evaluated on several metrics, as discussed below, including their predictive performance based on the test data sets, and also on data by sex and race/ethnicity in the US.

\subsection{Evaluation metrics}

In order to compare candidate models for ${ }_{1} a_{0}$ and evaluate their performance, several different evaluation metrics were used. First, we calculated the mean squared error (RMSE), which is defined as

$$
R M S E=\sqrt{\frac{\sum_{i=1}^{N}\left({ }_{1} a_{0}(i)-{ }_{1} \hat{a}_{0}(i)\right)^{2}}{N}}
$$

where ${ }_{1} a_{0}(i)$ is the $i$ th observed ${ }_{1} a_{0}$ value and ${ }_{1} \hat{a}_{0}(i)$ is the corresponding fitted value for all $N$ observations $i=1, \ldots, N$.

The RMSE was calculated on three different data sets for each of the three models (the ratio, piecewise, and AK model) under consideration:

1. The training data set, i.e. the HMD data on which the model was fitted, which comprises all available data (as described above) with $20 \%$ excluded. This is referred to the in-sample RMSE and reflects the goodness of model fit within the dataset that was used for estimation. 
2. The test data set, which is the $20 \%$ of HMD data that was excluded above. This is referred to the out-of-sample RMSE and reflects the model fit on a 'new' dataset.

3. The Andreev and Kingkade (AK) data set, i.e. the set of HMD data that was used by Andreev and Kingkade (2015). ${ }^{9}$ This was included to ensure that the piecewise estimates in Andreev and Kingkade (2015) and our piecewise estimates were as comparable as possible.

As an additional validation exercise, we also calculated the RMSE for the ratio and piecewise models based on ${ }_{1} a_{0}$ values calculated from the US microdata discussed in Section 2.1.

In addition to evaluating models based on in- and out-of-sample RMSE, we also used approximate leave-one-out cross-validation (LOO-CV) to assess relative model performance of the piecewise linear and the ratio model. LOO-CV refers to the technique of leaving out one data observation (the $i$ th data point), refitting the model to the new dataset with everything except point $i$, and evaluating the ability of the model to predict point $i$. This process can be repeated $N$ times, leaving out each of the $i$ points and then assessing predictive power each time. In practice, fitting each model $N$ times is computationally inefficient, and so we used approximate LOO-CV using Pareto smoothed importance sampling (Vehtari, Gelman, and Gabry 2017). This was done using the 100 package in $\mathrm{R}$ (Vehtari et al. 2020). Approximate LOO-CV gives an estimate for the expected log predictive density (ELPD). The higher the ELPD, the better the model fit.

Code and materials to reproduce our analysis can be found at https://github.com/ MJAlexander/a0-competing-effects.

\footnotetext{
${ }^{9}$ As discussed above, we used similar criteria for data inclusion as Andreev and Kingkade. However, a great deal of new data has been added to HMD since Andreev and Kingkade conducted their analysis, in early 2011, so their data set comprises only a subset of our data.
} 


\section{Results}

Table 4 shows the estimated coefficients for Equations 1 and 2 for various sex and data combinations. The first two rows show the estimates from Andreev and Kingkade (2015) for reference. The third and fourth rows show the results of estimation of the piecewise model fit to a dataset that is the same as was used by Andreev and Kingkade (2015). These results suggest that our estimates are very similar, which is encouraging given our estimation approach is quite different. The next three rows show results of the same piecewise model that has been fitted to all available HMD data. Notice that we also estimate this model for both sexes combined; this was motivated by the fact that, based on looking at the $95 \%$ uncertainty bounds of the sex-specific estimates, there is generally no significant difference between estimates for males and females. The coefficient estimates are slightly different to the AK data case, which perhaps is not surprising given the addition of many more data points at increasingly lower mortality conditions.

[Table 4 about here.]

The last three rows of Table 4 show the coefficient estimates for females, males and both sexes combined for the ratio model (shown in Equation 1). The sign of the coefficient estimates of the ratio model suggest that as infant mortality rises, so does ${ }_{1} a_{0}$, but as the fraction of under-five mortality that is attributed to infant mortality rises, ${ }_{1} a_{0}$ falls. The coefficient

estimates on infant mortality $\left(\hat{\beta}_{1}\right)$ are $1.5-1.9$, which suggests that for every 1 per 100 increase in infant mortality, ${ }_{1} a_{0}$ will increase by $0.015-0.02$ years, or somewhere between 5 to 7 days, holding the ratio constant. The coefficient estimates on the ratio $\left(\hat{\beta_{2}}\right)$ suggest that, as the ratio increases by $0.1,{ }_{1} a_{0}$ decreases by $0.03-0.04$ years, around $11-15$ days, holding infant mortality constant. 


\subsection{Model evaluation}

Table 5 shows in-sample and out-of-sample root mean squared error (RMSE) for each model, for each sex and for both sexes together. The smaller the RMSE, the better the model fit. Note that for the estimates from AK data, we can only calculated RMSE for either males or females, because estimates were not provided for both sexes combined. In general, the ratio model RMSEs are the smallest for all sexes, suggesting the fit of this model is superior to both the training (in-sample) and test (out of sample) data sets. The RMSE 'gain' going from the piecewise model to the ratio model is largest in the out-of-sample contexts, suggesting that the ratio model performs relatively well in predicting ${ }_{1} a_{0}$ based on new data.

[Table 5 about here.]

Finally, we can compare the expected log predictive density (ELPD) for the piecewise model and ratio model across all sex combinations. The higher the ELPD, the better the model's predictive ability. Table 6 shows the difference in ELPD (piecewise minus ratio) and the standard error for that difference for all sex combinations. The results suggest that in all cases, the ratio model has a higher ELPD (i.e. the difference is negative), although the difference is not significant in the male case.

\section{[Table 6 about here.]}

\subsubsection{Evaluation on US data}

One final model evaluation exercise involved calculating the accuracy of the ratio and piecewise models when estimating ${ }_{1} a_{0}$ for the US population by sex and race/ethnicity, where the microdata are available so the true value of ${ }_{1} a_{0}$ is known. Table 7 shows the RMSE calculated by race and sex for each of the models (the estimated values from the ratio model were calculated based on the coefficient estimates using both sexes combined). The ratio model outperforms the piecewise model in all cases. 
[Table 7 about here.]

\section{Discussion}

This paper increases our understanding of how and why length of life varies with other forms of inequality. We illustrate that higher infant mortality tends to raise ${ }_{1} a_{0}$, while a rising share of births that are premature tends to lower it. Thus, at a given level of infant mortality, a population with more premature birth will have a lower ${ }_{1} a_{0}$. This paradox explains the observed similarity of ${ }_{1} a_{0}$ among U.S.-born children of black and white non-Hispanic mothers; while children of black mothers have higher mortality, thus implying a longer ${ }_{1} a_{0}$, they also have higher rates of prematurity, implying a shorter one. In the U.S. case, the prematurity disadvantage outweighs the mortality disadvantage, resulting in a shorter ${ }_{1} a_{0}$ for infants born to black mothers.

We believe this is an important descriptive finding; it illustrates that the classic association of shorter ${ }_{1} a_{0}$ with better mortality conditions does not hold at low levels of mortality, where

the role of prematurity is greater. As medical advances have arisen that have tended both to lower infant mortality and to increase prematurity by allowing the survival of infants who would previously have been counted as stillbirths, the mechanisms that underlie infant survival and mortality have changed, disrupting well-known demographic patterns such as the Bourgeois-Pichat theory. Put simply, demographers must update their models to reflect this.

Our findings on racial disparities in the length of life for infants who die invite further exploration - for example, why is it that, as we have shown, length of life is drastically longer for premature black infants who die than for premature white ones, while for fullterm infants, the difference in length of life is minor? Work in medicine, public health and demography to explain the early mortality advantage among premature black infants is one avenue for this work, and has huge potential population health benefits. On the other side, 
there may exist patterns by cause of death that explain the disparity in length of life and indicate potential public health interventions to help curb the later infant mortality among premature black infants.

Finally, our findings on length-of-life patterns in the U.S. context — the fact that prematurity rates are so much higher for black infants that they completely swamp the effect on ${ }_{1} a_{0}$ of higher black late-in-infancy mortality — illustrate the extent of the U.S. premature birth crisis and its enormous role in the black infant mortality disadvantage. Further research in this area is crucial, as premature birth remains poorly understood; is a key point of interface between adult and child health; and poses a significant challenge for population health.

In the second half of the paper, we used this descriptive finding to motivate the formulation of a new approximation for ${ }_{1} a_{0}$ in low mortality conditions. What is the purpose of increasing the accuracy of ${ }_{1} a_{0}$ estimates? Small improvements in this measure are of relatively little importance for the life table (e.g., for the calculation of $e_{0}$ ); especially at low levels of mortality, the contribution of person-years lived by those who die within the first year of life to total person-years lived is very small, and the fact that a different ${ }_{1} a_{0}$ formula adjusts their modeled average length of life by mere days makes the effect smaller still. Nonetheless, accuracy is desirable; and, as well as illustrating an improvement in a purely statistical sense, we believe this model is an improvement because it is based on substantive reasoning rather than a mathematical function.

Furthermore, though it may not affect $e_{0}$ and other life table summary measures, the age pattern of infant mortality itself matters. Our framework explains and eliminates a portion of the previously unaccounted-for variation in ${ }_{1} a_{0}$ at low levels of mortality, and offers a new measure for differentiating between societies with very similar levels of infant mortality, but different patterns; as an illustrative example, one society at a given mortality level may have relatively low prematurity and a higher rate of deaths later in infancy from external causes, while another has high prematurity and fewer deaths later in infancy. The implications 
of these two patterns of death for public health policy and population wellbeing are very different.

The effectiveness of using the ratio of infant to under-five mortality as a proxy for prematurity in this application is also an important finding, and suggests a possible avenue for exploring an important phenomenon for which we have very little data. It also suggests that with more data on premature birth, ${ }_{1} a_{0}$ could be modeled with even greater accuracy.

A major limitation of this study is the restriction of the analysis to low-mortality contexts. The data and model estimation presented in this paper considered populations with infant mortality rates of less than 80 per 1,000, as data from HMD is largely limited to low-mortality populations. While it is likely that prematurity has the largest effect in populations with advanced medical systems - a requirement for the survival of premature infants - its effect in moderate and high-mortality contexts could also be important, and remains unknown. Future work could explore the relationship between ${ }_{1} a_{0}$, infant mortality and the ratio of infant to under-five mortality in a range of different mortality contexts, using, for example, data from Demographic and Health Surveys as well as child mortality estimates from UNIGME.

\section{Acknowledgments}

The authors would like to thank the anonymous reviewers for their helpful comments on previous drafts. 


\section{A Mortality and prematurity 2008-2012}

The tables below show mortality rates and prematurity rates for the non-Hispanic white and black populations for the United States for the years 2008-2012.

[Table 8 about here.]

[Table 9 about here.]

[Table 10 about here.] 


\section{B HMD data used}

The table below lists the countries and years included in the model estimation process.

[Table 11 about here.] 


\section{Details on simulation}

\section{C.1 Goals}

The goal of the simulation exercise is to see how ${ }_{1} a_{0}$ varies with changes in 1) the overall mortality risk and 2) the share of births that are premature. In order to do this, we need to first obtain a set of hazards for full-term births a preterm births. We then need to obtain a set of simulated death times based on these hazards under the conditions of 1) and 2).

\section{C.2 Estimating hazards}

In order to simulate infant death times under varying mortality risk and prematurity conditions, an expression for the hazard of dying at a particular time $t$ is required. However, the shape of the distribution of infant death times is non-parametric and thus not well represented by standard parametric distributions like the Exponential or Weibull.

As such, in order to obtain estimates for the hazards over time, we used piecewise constant hazard $(\mathrm{PCH})$ models. We assume exponential hazards within each piece, and split the first year at days $1-7,14,28,60,90,180$, and 365 . It can be shown that piece-wise exponential proportional hazards model is equivalent to a Poisson log-linear model where the death indicator is the response and the log of exposure time enters as an offset. We fit these models in $\mathrm{R}$ using the glm function separately for all full-term births and all preterm births in the 2012 U.S. dataset. The resulting regression estimates give the log-hazards in each of the separate intervals, which can then be used for simulation.

\section{C.3 Piecewise exponential survival function}

Define the set of time intervals to be $0=\tau_{0}<\tau_{1}<\ldots \tau_{m}<\tau_{m+1}$. The piecewise constant hazard function is 


$$
h(t)=h_{0} \sum_{l=0}^{m} g_{l} I_{l}(t)
$$

where $h_{0}$ is the baseline hazard, $g_{l}$ are the relative hazards and $I(t)$ is an indicator function which is equal to 1 if $\tau_{l} \leq t<\tau_{l+1}$ and 0 otherwise.

The survival function is

$$
S(t)=\exp (-H(t))=\exp \left(-h_{0} \sum_{l=0}^{m} g_{l} \int_{0}^{t} I_{l}(s) d s\right)
$$

The piecewise survival function for interval if $\tau_{i} \leq t<\tau_{i+1}$ is

$$
S_{i}(t)=\exp \left(-h_{0}\left(\sum_{l=0}^{i-1} g_{l}\left(\tau_{l+1}-\tau_{l}\right)+g_{i}\left(t-t_{i}\right)\right)\right)
$$

Solving for $t$ implies

$$
t=\tau_{i}-\frac{\log \left(S_{i}(t)\right)}{h_{0} g_{i}}-\frac{1}{g_{i}} \sum_{l=0}^{i-1} g_{l}\left(\tau_{l+1}-\tau_{l}\right)
$$

As $\tau_{i} \leq t<\tau_{i+1}$ this gives us two conditions:

$$
\begin{aligned}
& \log \left(S_{i}(t)\right) \leq-h_{0} \sum_{l=0}^{i-1} g_{l}\left(\tau_{l+1}-\tau_{l}\right) \\
& \log \left(S_{i}(t)\right)>\sum_{l=0}^{i} g_{l}\left(\tau_{l+1}-\tau_{l}\right)
\end{aligned}
$$

\section{C.4 Simulation framework}

We have an analytical form for the survival function $S$, which means there is also an analytical form for the cumulative distribution function $F=1-S$. We used inverse transform sampling and Equations 3-5 above to generate samples from the death distributions specified by a set 
of piecewise hazards estimated using Poisson regression. To reiterate, the two simulation scenarios were:

- Scenario 1: Vary the risk of infant mortality of a population with similar infant mortality to the full-term birth population in the U.S. Risk multipliers simulated were between $[0.1,3]$ in increments of 0.1 .

- Scenario 2: Vary the share of premature births in a population which has similar hazard profiles of pre- and full-term births in the U.S. Shares considered were between $[0,0.5]$ in increments of 0.1 .

The following simulation process was used:

1. Set the time intervals, the baseline hazard, and all relative hazards. For Scenario 1, the hazards come from the full-term birth estimation. For Scenario 2, both the hazards from full- and preterm births are used. For every iteration in each scenario (i.e. each risk multiplier and each share $s$ )

2. Set the size of the sub-population. For Scenario 1 this was 10,000. For Scenario 2 this was $10,000 * s$ preterm births and $10,000 *(1-s)$ full-term births.

3. Draw a uniformly $(0,1)$ distributed random variable $\mathrm{S}=1-\mathrm{F}$. (For Scenario 2, two random variables are drawn.)

4. Determine the right interval using the conditions in Equations 4 and 5.

5. Compute the random time t using Equation 3.

6. Combine the computed random times to one file.

7. Repeat this process 1,000 times. 
Once the set of 1,000 simulation times are obtained, the mean age of death ${ }_{1} a_{0}$ was calculated for each simulation. The mean, 2.5th and 97.5th quantiles of ${ }_{1} a_{0}$ were then calculated.

Code to reproduce this simulation can be found at https://github.com/MJAlexander/a0competing-effects. 


\section{References}

Alhusen, Jeanne L., Kelly Bower, Elizabeth Epstein, and Phyllis Sharps. 2016. "Racial Discrimination and Adverse Birth Outcomes: An Integrative Review." Journal of Midwifery \& Women's Health 61 (6): 707-20. https://doi.org/10.1111/jmwh.12490.

Andreev, Evgeny M., and W. Ward Kingkade. 2015. "Average Age at Death in Infancy and Infant Mortality Level: Reconsidering the Coale-Demeny Formulas at Current Levels of Low Mortality." Demographic Research 33 (13): 363-90. https://doi.org/10.4054/ DemRes.2015.33.13.

Bediako, Phylicia T., Rhonda BeLue, and Marianne M. Hillemeier. 2015. "A Comparison of Birth Outcomes Among Black, Hispanic, and Black Hispanic Women." Journal of Racial and Ethnic Health Disparities 2 (4): 573-82. https://doi.org/10.1007/s40615-015-0110-2.

Blencowe, Hannah, Simon Cousens, Mikkel Z Oestergaard, Doris Chou, Ann-Beth Moller, Rajesh Narwal, Alma Adler, et al. 2012. "National, Regional, and Worldwide Estimates of Preterm Birth Rates in the Year 2010 with Time Trends Since 1990 for Selected Countries: A Systematic Analysis and Implications." The Lancet 379 (9832): 2162-72. https://doi.org/10.1016/S0140-6736(12)60820-4.

Bourgeois-Pichat, Jean. 1951a. "La Mesure de La Mortalité Infantile. I. Principes Et méthodes." Population (French Edition) 6 (2): 233-48. https://doi.org/10.2307/1524151.

—. 1951b. "La Mesure de La Mortalité Infantile: II. Les Causes de décès." Population (French Edition) 6 (3): 459-80. https://doi.org/10.2307/1523958.

Callaghan, William M, Marian F MacDorman, Sonja A Rasmussen, Cheng Qin, and Eve M Lackritz. 2006. "The Contribution of Preterm Birth to Infant Mortality Rates in the United States." Pediatrics 118 (4): 1566-73.

Carpenter, Bob, Andrew Gelman, Matthew D Hoffman, Daniel Lee, Ben Goodrich, Michael Betancourt, Marcus Brubaker, Jiqiang Guo, Peter Li, and Allen Riddell. 2017. "Stan: 
A Probabilistic Programming Language." Journal of Statistical Software 76 (1).

Centers for Disease Control and Prevention National Center for Health Statistics. 2020. "Natality Public-Use Data on CDC WONDER Online Database." Available at: https: //wonder.cdc.gov/wonder.

Coale, Ansley J., Paul George Demeny, and Barbara Vaughan. 1983. Regional Model Life Tables and Stable Populations. 2nd ed. Studies in Population. New York: Academic Press.

Ely, Danielle M., and Anne K. Driscoll. 2019. "Infant Mortality in the United States, 2017: Data From the Period Linked Birth/Infant Death File." 68: 10. U.S. Department of Health; Human Services, Centers for Disease Control; Prevention.

Galley, Chris, and Robert Woods. 1999. "On the Distribution of Deaths During the First Year of Life." Population: An English Selection 11: 35-59. http://www.jstor.org/stable/ 2998689.

Hummer, Robert A., Monique Biegler, Peter B. De Turk, Douglas Forbes, W. Parker Frisbie, Ying Hong, and Starling G. Pullum. 1999. "Race/Ethnicity, Nativity, and Infant Mortality in the United States." Social Forces 77 (3): 1083-1117. https://doi.org/10. $2307 / 3005972$.

James, Gareth, Daniela Witten, Trevor Hastie, and Robert Tibshirani. 2013. An Introduction to Statistical Learning. Vol. 112. Springer.

Keyfitz, Nathan, and Wilhelm Flieger. 1971. Population: Facts and Methods of Demography. San Francisco: W. H. Freeman.

Kramer, Michael R., and Carol R. Hogue. 2009. "What Causes Racial Disparities in Very Preterm Birth? A Biosocial Perspective." Epidemiologic Reviews 31: 84-98. https: //doi.org/10.1093/ajerev/mxp003.

MacDorman, Marian F. 2011. "Understanding Racial and Ethnic Disparities in U.S. Infant 
Mortality Rates," no. 74: 8.

Mathews, T. J., Marian F. MacDorman, and Fay Menacker. 2013. "Infant Mortality Statistics from the 2013 Period Linked Birth/Infant Death Data Set." https://doi.org/10.1037/ e558952006-001.

Mejía-Guevara, Iván, and Shripad Tuljapurkar. 2019. “'Early Rectangularization” of Under5 Mortality in Sub-Saharan Africa: Compression, Convergence, Inequality." bioRxiv, March, 591925. https://doi.org/10.1101/591925.

Mejı́a-Guevara, Iván, Wenyun Zuo, Eran Bendavid, Nan Li, and Shripad Tuljapurkar. 2019. "Age Distribution, Trends, and Forecasts of Under-5 Mortality in 31 Sub-Saharan African Countries: A Modeling Study." PLoS Medicine 16 (3). https://doi.org/10.1371/journal. pmed.1002757.

NBER. 2020. "Linked Birth/Infant Death Cohort Data." Available at: https://www.nber. org/research/data/linked-birthinfant-death-cohort-data.

Purisch, Stephanie E., and Cynthia Gyamfi-Bannerman. 2017. "Epidemiology of Preterm Birth." Seminars in Perinatology, Current Preterm Birth Prevention Strategies, 41 (7): 387-91. https://doi.org/10.1053/j.semperi.2017.07.009.

Riddell, Corinne A., Sam Harper, and Jay S. Kaufman. 2017. "Trends in Differences in US Mortality Rates Between Black and White Infants." JAMA Pediatrics 171 (9): 911-13. https://doi.org/10.1001/jamapediatrics.2017.1365.

Tucker, Janet, and William McGuire. 2004. "Epidemiology of Preterm Birth." BMJ 329 (7467): 675-78. https://doi.org/10.1136/bmj.329.7467.675.

Vehtari, Aki, Jonah Gabry, Mans Magnusson, Yuling Yao, Paul-Christian Bürkner, Topi Paananen, and Andrew Gelman. 2020. "Loo: Efficient Leave-One-Out Cross-Validation and WAIC for Bayesian Models." https://mc-stan.org/loo.

Vehtari, Aki, Andrew Gelman, and Jonah Gabry. 2017. "Practical Bayesian Model Evalu- 
ation Using Leave-One-Out Cross-Validation and WAIC." Statistics and Computing 27 (5): $1413-32$.

Wachter, Kenneth W. 2014. Essential Demographic Methods. Cambridge, Massachusetts: Harvard University Press.

WHO. 2018. "WHO Fact Sheet: Preterm Birth.” World Health Organization. https://www. who.int/news-room/fact-sheets/detail/preterm-birth. 


\section{List of Figures}

1 Proportion of infant deaths by five day intervals, United States, 2012 birth cohort. Data via National Center for Health Statistics National Vital Statistics System. Note differing y-axis scales. . . . . . . . . . . . . . . . . .

2 Estimated ${ }_{1} a_{0}$ and $95 \%$ confidence intervals for different mortality risk factors and shares of premature birth. Results from 1,000 simulations of 10,000 births with baseline hazards for pre- and full-term births fitted to the U.S. population

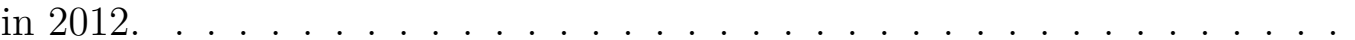

3 Relationship between the ratio of infant to under-five mortality and prematurity in the United States. . . . . . . . . . . . . . . . . . . .

4 Relationship between the ratio of infant to under-five mortality and ${ }_{1} a_{0}$ in low-mortality conditions. . . . . . . . . . . . . . . . . . 36 

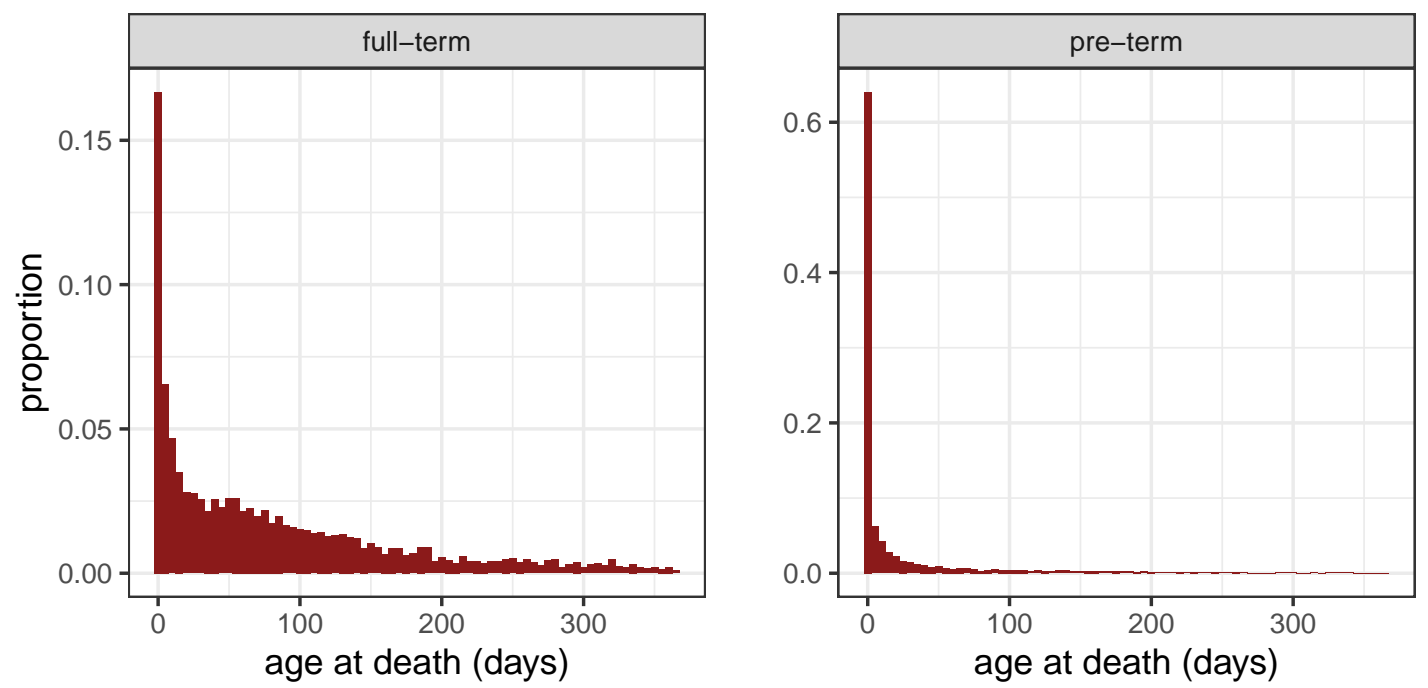

Figure 1: Proportion of infant deaths by five day intervals, United States, 2012 birth cohort. Data via National Center for Health Statistics National Vital Statistics System. Note differing $\mathrm{y}$-axis scales. 


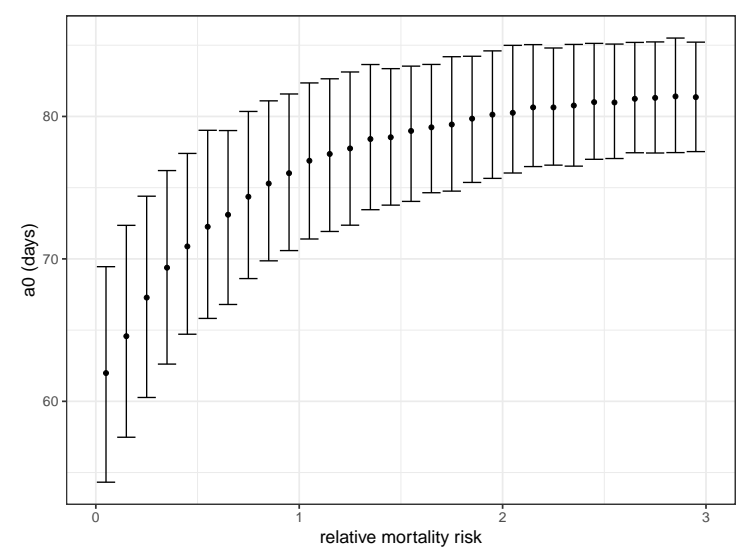

(a) Estimated ${ }_{1} a_{0}$ varying the mortality risk factors.

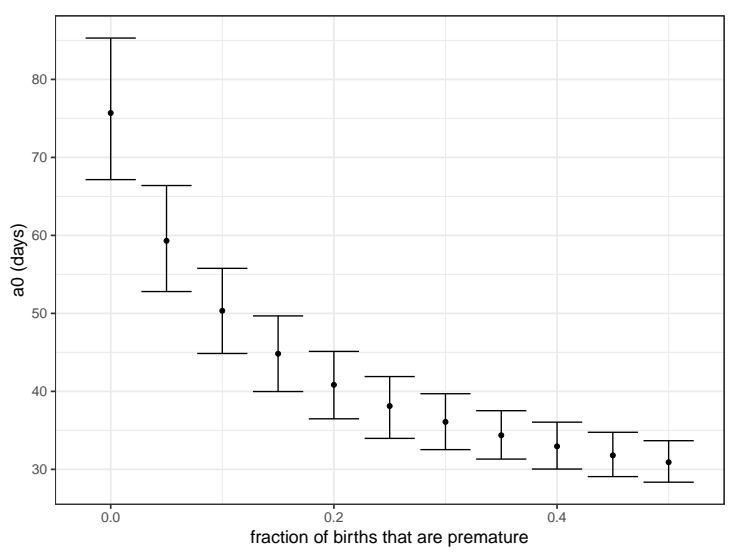

(b) Estimated ${ }_{1} a_{0}$ varying the share of premature births.

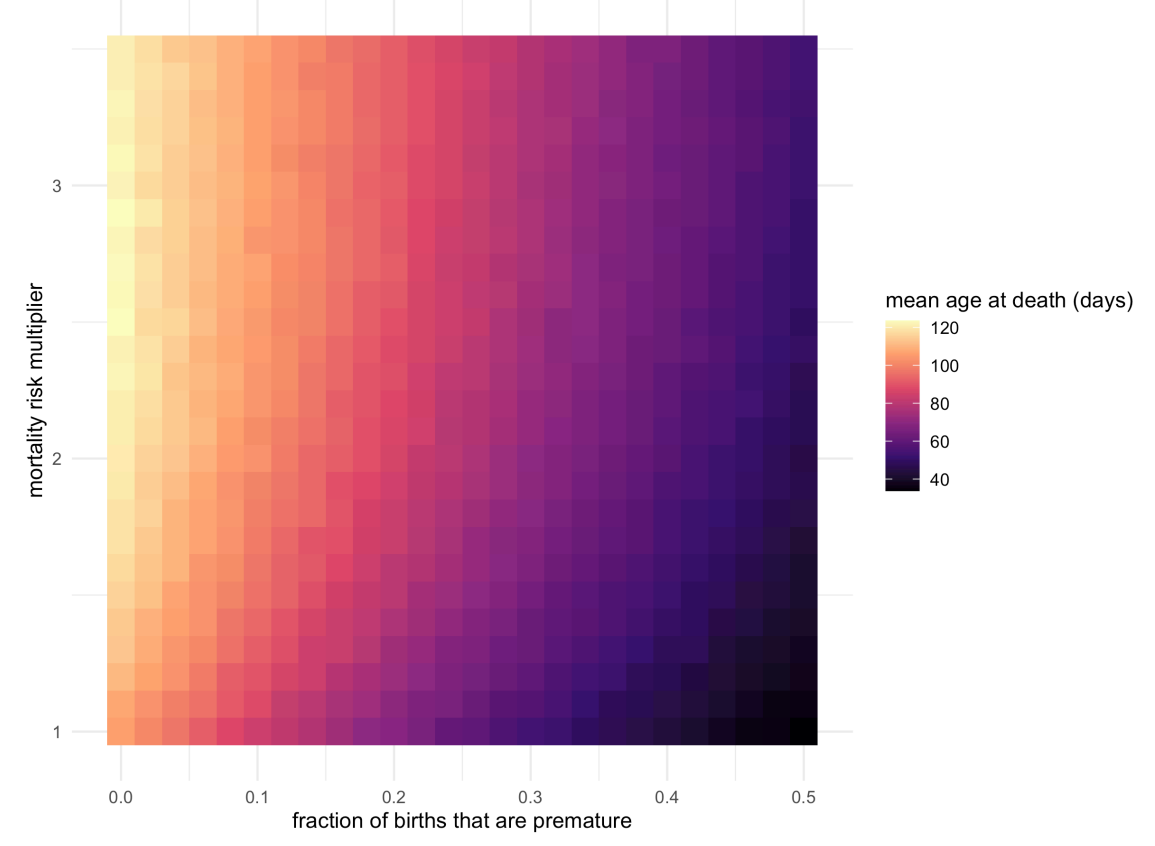

(c) Competing effects on average age of infant death. Horizontal axis shows increasing prematurity, vertical axis shows increasing overall mortality risk.

Figure 2: Estimated ${ }_{1} a_{0}$ and $95 \%$ confidence intervals for different mortality risk factors and shares of premature birth. Results from 1,000 simulations of 10,000 births with baseline hazards for pre- and full-term births fitted to the U.S. population in 2012. 


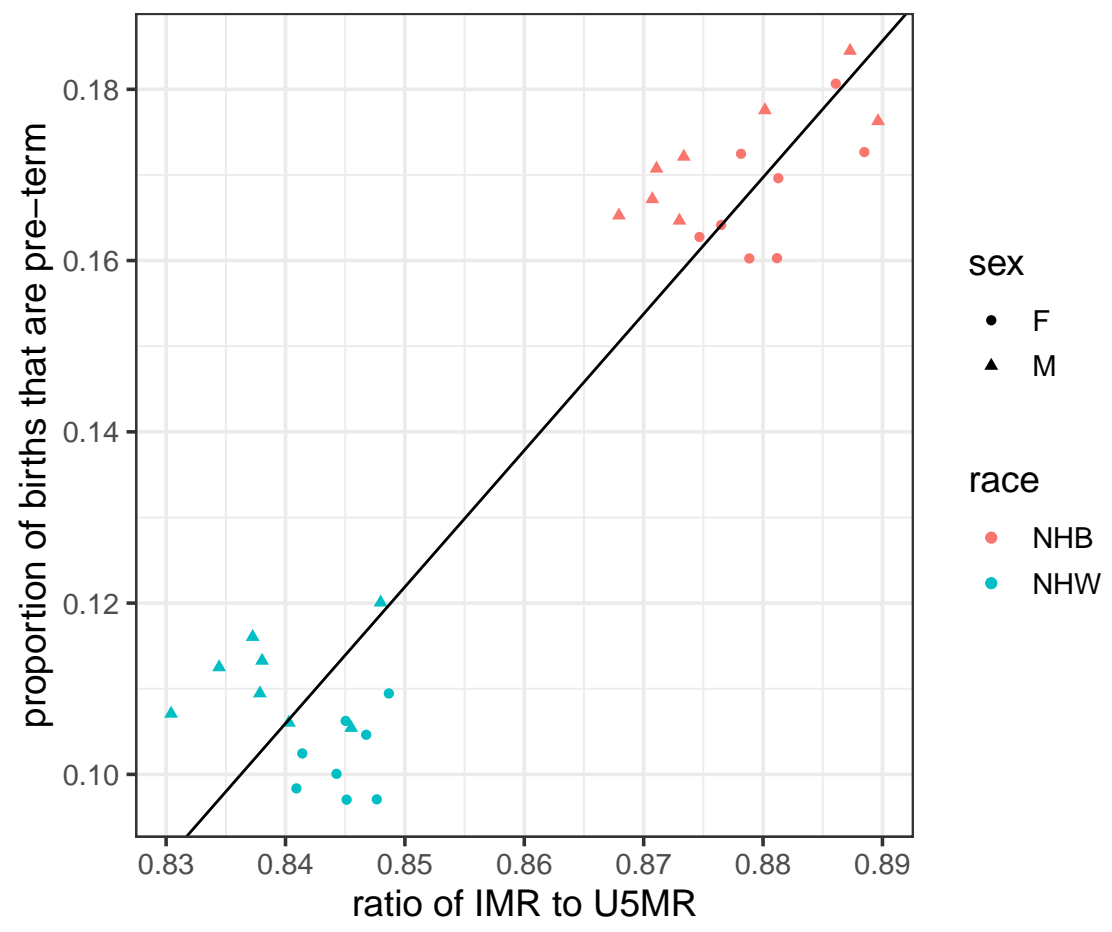

Figure 3: Relationship between the ratio of infant to under-five mortality and prematurity in the United States. 


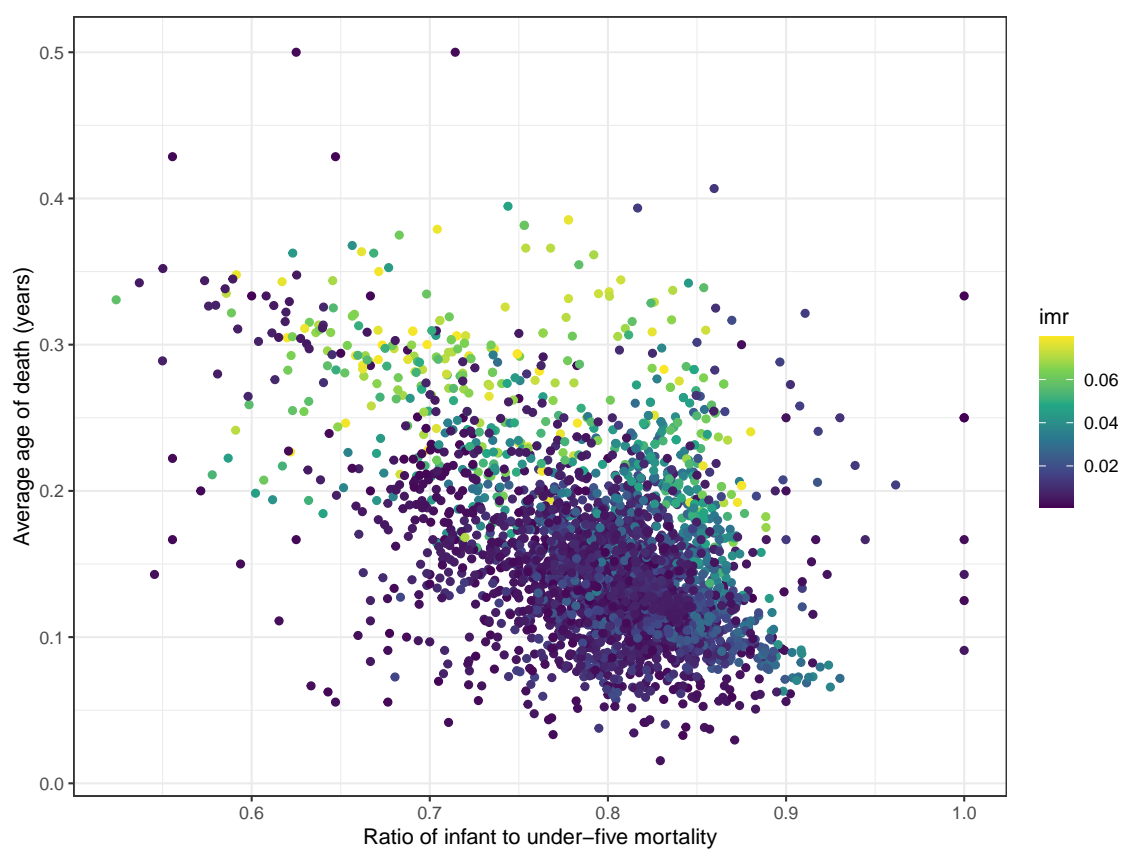

Figure 4: Relationship between the ratio of infant to under-five mortality and ${ }_{1} a_{0}$ in lowmortality conditions. 


\section{List of Tables}

1 Infant mortality rates per thousand for U.S. children of non-Hispanic black and non-Hispanic white mothers and the ratio between them . . . . . . . 38

2 Rates of preterm and full-term birth per 1000 U.S. live births to non-Hispanic black and non-Hispanic white mothers and the ratio between them . . . . . 39

3 Mean age at death (days) by gestational age at birth for U.S. infants born to non-Hispanic black and non-Hispanic white mothers and the ratio between them .............................. 40

4 Coefficient estimates (and 95\% credible intervals) for all model combinations 41

$5 \quad$ In- and out-of-sample root mean squared error for different models . . . . . 42

6 ELPD difference (piecewise - ratio) and standard error of difference for all sex combinations . . . . . . . . . . . . . . . . . . . 43

7 Root mean squared error of piecewise and ratio models fitted to US data by sex and race/ethnicity. . . . . . . . . . . . . . . . . 44

8 Mortality rates by age at death, race, and year . . . . . . . . . . . . 45

$9 \quad$ Rates of preterm and full-term birth per 1000 live births by race and year . . 46

10 Mortality rates by premature status, race, and year . . . . . . . . . . 47

11 Human Mortality Database populations and cohorts that meet criteria for inclusion in analysis. Note that some cohorts listed here are excluded based on the infant mortality rate cutoff of 80 per thousand (female) and 95 per thousand (male). . . . . . . . . . . . . . . . . . 


\begin{tabular}{|l|l|rrr|rrrr|}
\hline & & \multicolumn{3}{|c|}{ by age at death } & \multicolumn{4}{c|}{ by gestational age at birth } \\
& total & first week & neonatal & post-neonatal & extremely pre-term & very pre-term & later pre-term & full-term \\
\hline black & 11.00 & 5.94 & 7.30 & 3.70 & 363.18 & 38.86 & 9.01 & 3.54 \\
white & 4.98 & 2.60 & 3.26 & 1.72 & 365.32 & 38.90 & 8.65 & 2.09 \\
\hline ratio & 2.21 & 2.29 & 2.24 & 2.15 & 0.99 & 1.00 & 1.04 & 1.70 \\
\hline
\end{tabular}

Table 1: Infant mortality rates per thousand for U.S. children of non-Hispanic black and non-Hispanic white mothers and the ratio between them 


\begin{tabular}{|l|rrrr|}
\hline & \multicolumn{4}{|c|}{ Births (rates per thousand) } \\
\hline & extremely preterm & very preterm & later preterm & full-term \\
\hline black & 16.79 & 20.32 & 128.15 & 834.75 \\
white & 5.36 & 10.14 & 87.42 & 897.09 \\
\hline ratio & 3.13 & 2.00 & 1.47 & 0.93 \\
\hline
\end{tabular}

Table 2: Rates of preterm and full-term birth per 1000 U.S. live births to non-Hispanic black and non-Hispanic white mothers and the ratio between them 


\begin{tabular}{|l|r|rrrr|}
\hline & \multicolumn{5}{|c|}{ mean age at death (days) } \\
\hline & aggregate & extremely preterm & very preterm & later preterm & full term \\
\hline black & 40.87 & 14.21 & 41.73 & 68.26 & 84.97 \\
white & 45.29 & 9.93 & 31.53 & 51.05 & 82.85 \\
\hline ratio & 0.90 & 1.43 & 1.32 & 1.34 & 1.03 \\
\hline
\end{tabular}

Table 3: Mean age at death (days) by gestational age at birth for U.S. infants born to nonHispanic black and non-Hispanic white mothers and the ratio between them 


\begin{tabular}{|l|l|l|r|r|r|r|}
\hline Model & Equation & Sex & \multicolumn{1}{l|}{$\hat{\alpha}$} & \multicolumn{1}{|c|}{$\hat{\beta_{1}}$} & \multicolumn{1}{l}{$\hat{\beta}_{2}$} \\
\hline AK & 2 & $\mathrm{f}$ & 0.149 & -2.0867 & 4.1075 & 0.017 \\
AK & 2 & $\mathrm{~m}$ & 0.1493 & -2.0367 & 3.4994 & 0.0226 \\
piecewise (AK data) & 2 & $\mathrm{f}$ & $0.149[0.14,0.155]$ & $-1.984[-2.82,-1.093]$ & $4.856[3.87,5.695]$ & $0.014[0.01,0.016]$ \\
piecewise (AK data) & 2 & $\mathrm{~m}$ & $0.15[0.14,0.158]$ & $-2.162[-2.87,-1.588]$ & $4.647[4.03,5.41]$ & $0.019[0.02,0.021]$ \\
piecewise & 2 & $\mathrm{f}$ & $0.138[0.13,0.143]$ & $-0.913[-1.59,-0.328]$ & $4.249[3.62,4.976]$ & $0.017[0.01,0.019]$ \\
piecewise & 2 & $\mathrm{~m}$ & $0.14[0.14,0.145]$ & $-1.268[-1.74,-0.883]$ & $4.478[3.99,4.975]$ & $0.023[0.02,0.025]$ \\
piecewise & 2 & $\mathrm{~b}$ & $0.139[0.14,0.142]$ & $-1.004[-1.38,-0.647]$ & $4.297[3.92,4.708]$ & $0.021[0.02,0.022]$ \\
ratio & 1 & $\mathrm{f}$ & $0.405[0.37,0.436]$ & $1.975[1.87,2.084]$ & $-0.359[-0.4,-0.322]$ & - \\
ratio & 1 & $\mathrm{~m}$ & $0.42[0.39,0.454]$ & $1.551[1.43,1.669]$ & $-0.382[-0.42,-0.341]$ & - \\
ratio & 1 & $\mathrm{~b}$ & $0.426[0.4,0.447]$ & $1.749[1.67,1.834]$ & $-0.387[-0.41,-0.359]$ & - \\
\hline
\end{tabular}

Table 4: Coefficient estimates (and 95\% credible intervals) for all model combinations 


\begin{tabular}{|l|l|l|r|l|}
\hline Type & Sex & AK & piecewise & ratio \\
\hline in-sample & $\mathrm{f}$ & 0.038 & 0.038 & 0.037 \\
in-sample (AK data) & $\mathrm{f}$ & 0.035 & 0.038 & 0.036 \\
out of sample & $\mathrm{f}$ & 0.038 & 0.038 & 0.036 \\
insample & $\mathrm{m}$ & 0.036 & 0.036 & 0.036 \\
in-sample (AK data) & $\mathrm{m}$ & 0.042 & 0.038 & 0.038 \\
out of sample & $\mathrm{m}$ & 0.044 & 0.041 & 0.040 \\
in-sample & $\mathrm{b}$ & - & 0.038 & 0.037 \\
out of sample & $\mathrm{b}$ & - & 0.041 & 0.038 \\
\hline
\end{tabular}

Table 5: In- and out-of-sample root mean squared error for different models 


\begin{tabular}{|l|ll|}
\hline Sex & ELPD difference & SE difference \\
\hline $\mathrm{f}$ & -54.27 & 20.47 \\
$\mathrm{~m}$ & -10.96 & 21.67 \\
$\mathrm{~b}$ & -83.98 & 30.89 \\
\hline
\end{tabular}

Table 6: ELPD difference (piecewise - ratio) and standard error of difference for all sex combinations 


\begin{tabular}{|l|l|l|l|}
\hline Race & Sex & Model & RMSE \\
\hline NHB & F & ratio & 0.0090 \\
NHB & F & piecewise & 0.0409 \\
NHW & F & ratio & 0.0129 \\
NHW & F & piecewise & 0.0689 \\
NHB & M & ratio & 0.0080 \\
NHB & M & piecewise & 0.0355 \\
NHW & M & ratio & 0.0139 \\
NHW & M & piecewise & 0.0710 \\
\hline
\end{tabular}

Table 7: Root mean squared error of piecewise and ratio models fitted to US data by sex and race/ethnicity. 


\begin{tabular}{|l|rrrrr|}
\hline & 2008 & 2009 & 2010 & 2011 & 2012 \\
\hline & \multicolumn{5}{|c|}{ infant } \\
\hline black & 12.34 & 12.03 & 11.32 & 11.07 & 11.00 \\
\hline white & 5.40 & 5.19 & 5.06 & 4.98 & 4.98 \\
\hline ratio & 2.29 & 2.32 & 2.24 & 2.22 & 2.21 \\
\hline \multicolumn{5}{|c|}{ first-week } & mortality rate \\
\hline black & 6.48 & 6.33 & 5.85 & 6.03 & 5.94 \\
\hline white & 2.71 & 2.59 & 2.56 & 2.57 & 2.60 \\
\hline ratio & 2.39 & 2.44 & 2.28 & 2.35 & 2.29 \\
\hline \multicolumn{5}{|c|}{ neonatal } & mortality rate \\
\hline black & 8.07 & 7.93 & 7.28 & 7.43 & 7.30 \\
\hline white & 3.44 & 3.32 & 3.29 & 3.25 & 3.26 \\
\hline ratio & 2.35 & 2.39 & 2.21 & 2.29 & 2.24 \\
\hline & \multicolumn{5}{|c}{ post-neonatal mortality rate } \\
\hline black & 4.27 & 4.09 & 4.04 & 3.64 & 3.70 \\
\hline white & 1.96 & 1.87 & 1.77 & 1.74 & 1.72 \\
\hline ratio & 2.18 & 2.19 & 2.29 & 2.10 & 2.15 \\
\hline
\end{tabular}

Table 8: Mortality rates by age at death, race, and year 


\begin{tabular}{|l|rrrrr|}
\hline & 2008 & 2009 & 2010 & 2011 & 2012 \\
\hline & \multicolumn{5}{|c|}{ extremely preterm } \\
\hline black & 17.19 & 17.43 & 16.89 & 16.99 & 16.79 \\
\hline white & 5.41 & 5.36 & 5.44 & 5.24 & 5.36 \\
\hline ratio & 3.18 & 3.25 & 3.10 & 3.25 & 3.13 \\
\hline \multicolumn{5}{|c|}{ very preterm } \\
\hline black & 21.24 & 21.25 & 20.99 & 20.58 & 20.32 \\
\hline white & 10.58 & 10.38 & 10.36 & 10.21 & 10.14 \\
\hline ratio & 2.01 & 2.05 & 2.03 & 2.02 & 2.00 \\
\hline & \multicolumn{5}{|c|}{ later preterm } \\
\hline black & 137.00 & 135.98 & 133.30 & 130.17 & 128.15 \\
\hline white & 95.39 & 93.43 & 91.89 & 89.56 & 87.42 \\
\hline ratio & 1.44 & 1.46 & 1.45 & 1.45 & 1.47 \\
\hline & \multicolumn{5}{|c|}{ full term } \\
\hline black & 824.57 & 825.34 & 828.82 & 832.26 & 834.75 \\
\hline white & 888.62 & 890.82 & 892.30 & 895.00 & 897.09 \\
\hline ratio & 0.93 & 0.93 & 0.93 & 0.93 & 0.93 \\
\hline
\end{tabular}

Table 9: Rates of preterm and full-term birth per 1000 live births by race and year 


\begin{tabular}{|l|rrrrr|}
\hline & 2008 & 2009 & 2010 & 2011 & 2012 \\
\hline \multicolumn{5}{|c|}{ extremely preterm } \\
\hline black & 398.48 & 384.46 & 366.24 & 362.18 & 363.18 \\
\hline white & 387.33 & 376.18 & 369.73 & 369.00 & 365.32 \\
\hline ratio & 1.03 & 1.02 & 0.99 & 0.98 & 0.99 \\
\hline \multicolumn{5}{|c|}{ very preterm } \\
\hline black & 46.62 & 45.77 & 44.10 & 40.36 & 38.86 \\
\hline white & 40.26 & 41.40 & 37.39 & 41.58 & 38.90 \\
\hline ratio & 1.16 & 1.11 & 1.18 & 0.97 & 1.00 \\
\hline \multicolumn{5}{|c|}{ later preterm } \\
\hline black & 10.46 & 9.92 & 9.67 & 9.88 & 9.01 \\
\hline white & 8.84 & 8.18 & 8.30 & 8.26 & 8.65 \\
\hline ratio & 1.18 & 1.21 & 1.16 & 1.20 & 1.04 \\
\hline & \multicolumn{5}{|c|}{ full term } \\
\hline black & 3.72 & 3.64 & 3.53 & 3.36 & 3.54 \\
\hline white & 2.29 & 2.22 & 2.13 & 2.11 & 2.09 \\
\hline ratio & 1.63 & 1.64 & 1.66 & 1.60 & 1.70 \\
\hline
\end{tabular}

Table 10: Mortality rates by premature status, race, and year 


\begin{tabular}{|l|l|l|}
\hline Population & HMD abbreviation & Cohorts \\
\hline Australia & AUS & $1990-2015$ \\
\hline Austria & AUT & $1971-2016$ \\
\hline Belgium & BEL & $1956-1957,1961-2016$ \\
\hline Bulgaria & BGR & $2009-2016$ \\
\hline Canada & CAN & $1950-2009$ \\
\hline Croatia & HRV & $2001-2017$ \\
\hline Czech Republic & CZE & $1950-2017$ \\
\hline Denmark & DNK & $1921-2018$ \\
\hline England and Wales & GBRTENW & $1914-1949$ \\
\hline Estonia & EST & $1992-2016$ \\
\hline Finland & FIN & $1917-2017$ \\
\hline France (civilian population) & FRACNP & $1907-2016$ \\
\hline Germany (East) & DEUTE & $1956-2016$ \\
\hline Germany (West) & DEUTW & $1956-2016$ \\
\hline Hungary & HUN & $1950-2016$ \\
\hline Italy & ITA & $1915-2016$ \\
\hline Japan & JPN & $1947-2017$ \\
\hline Netherlands & NLD & $1850-1998$ \\
\hline New Zealand & NZL_NP & $1980-2012$ \\
\hline Norway & NOR & $1980-2017$ \\
\hline Portugal & PRT & $1980-2017$ \\
\hline Slovakia & SVK & $1965-2016$ \\
\hline Slovenia & SVN & $1983-2016$ \\
\hline Spain & ESP & $1975-2017$ \\
\hline Sweden & SWE & $1901-2017$ \\
\hline Switzerland & CHE & $1880-2017$ \\
\hline United States of America & USA & $1959-2016$ \\
\hline & & \\
\hline & & \\
\hline & & \\
\hline
\end{tabular}

Table 11: Human Mortality Database populations and cohorts that meet criteria for inclusion in analysis. Note that some cohorts listed here are excluded based on the infant mortality rate cutoff of 80 per thousand (female) and 95 per thousand (male). 